\title{
Was LIGO's Gravitational Wave Detection a False Alarm?
}

\author{
Policarpo Yōshin Ulianov ${ }^{1}$, Xiaochun $\mathrm{Mei}^{2}$, Ping $\mathbf{Y u}^{3}$ \\ ${ }^{1}$ Equalix Tecnologia LTDA, Florianópolis, SC, Brazil \\ ${ }^{2}$ Institute of Innovative Physics, Fuzhou, Fujian, China \\ ${ }^{3}$ Cognitech Calculating Technology Institute, Los Angeles, CA, USA \\ Email: policarpoyu@gmail.com, ycwlyjs@yeah.net, yupingpingyu@yahoo.com
}

How to cite this paper: Ulianov, P.Y., Mei, X.C. and Yu, P. (2016) Was LIGO's Gravitational Wave Detection a False Alarm? Journal of Modern Physics, 7, 1845-1865.

http://dx.doi.org/10.4236/jmp.2016.714164

Received: August 11, 2016

Accepted: October 14, 2016

Published: October 17, 2016

Copyright $\odot 2016$ by authors and Scientific Research Publishing Inc. This work is licensed under the Creative Commons Attribution International License (CC BY 4.0).

http://creativecommons.org/licenses/by/4.0/

\section{(c) (i) Open Access}

\begin{abstract}
This article presents a new type of whitening filter (allowing the "passing" of some noise sources) applied to process the data recorded in LIGO's GW150914 and GW151226 events. This new analysis shows that in the GW150914 event, the signals from the collision of two black holes are very similar to the $32.5 \mathrm{~Hz}$ noise sources observed in both of LIGO's detectors. It also points out that these $32.5 \mathrm{~Hz}$ noise sources are powered by a $30 \mathrm{~Hz}$ sub harmonic, coming from the $60 \mathrm{~Hz}$ power system. In the GW1226 event, the same analysis points out that the NR template is very similar to the $120 \mathrm{~Hz}$ noise source. Therefore, the signals recorded in these events were probably generated by some small changes with the $60 \mathrm{~Hz}$ frequency in the US power grid. This can be caused, for example, by a power variation in the DC link, which can appear in both detectors in the same $10 \mathrm{~ms}$ time window. As this kind of power grid occurrence did not change the voltage levels, it may have gone unnoticed by LIGO's electrical power supply's monitoring system.
\end{abstract}

\section{Keywords}

Gravitational Waves Detection, LIGO, Laser Interferometer Gravitational Wave Observatory

\section{Introduction}

The first experiment, with the objective of detecting gravitational waves, was conducted in the 1960's by Joseph Weber [1] at the University of Maryland. Weber used large cylinders of aluminum that supposedly vibrated in response to a passing gravitational wave, an approach that did not work.

Until 2015, experimental attempts to detect gravitational waves were conducted for 
over 55 years without any results. Today, LIGO's (Laser Interferometer GravitationalWave Observatory) experiment is the largest and most expensive ever built for detecting gravitational waves, comprising two interferometers, one located in Hanford, Washington, and the other in Livingston, Louisiana.

LIGO's initial search (from 2002 to 2010) did not detect any gravitational waves, clear evidence that there was something wrong with the first version of LIGO's system. In September 2015, LIGO completed a 5-year overhaul of their system at a total cost of $\$ 620$ million, making it ten times more accurate and renaming it “Advanced LIGO”.

On September 14th, 2015, shortly after “Advanced LIGO” was put into operation, it recorded the GW150914 event. After five months of analysis on the GW150914 event's recorded data, a job that involves about four hundred physicists from a dozen institutions, the LIGO team reported [2] the detection of its first gravitational wave. The signals related to the detection are shown in Figure 1.

On December 26th, 2015, a second gravitational wave detection was reported by the LIGO team [3] from the GW151226 event.

For these authors, the LIGO system operated more than a decade without obtaining any results due to some theoretical and practical problems in the basis of this experiment. Detailed information about these problems can be found in articles [4] and [5], presented in summarized form in Section 2 of this article.

The theoretical factors pointed out in Section 2 can generate plenty of controversy, thus, the theoretical discussion can go on and on.

However, the important fact was that the data recorded by LIGO was made available on the internet along with the program codes used for processing it. This allowed a more thorough assessment of the "gravitational waves" recorded by LIGO, which, as presented in Sections 3 and 4, had a strange resemblance to some noise signals coming from the detectors.

It is important to observe that the noise levels in both of LIGO's detectors are a hundred times bigger than the expected levels of the gravitational wave signals. Thus, the

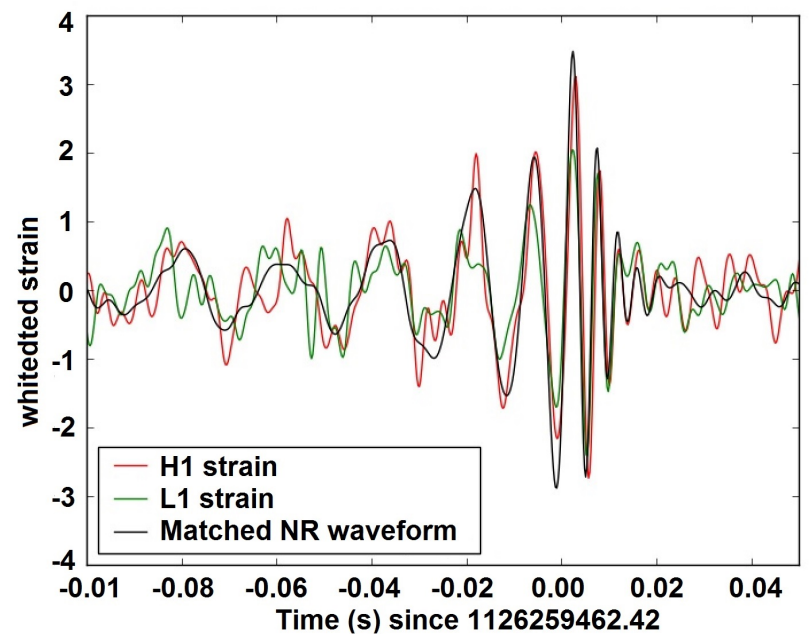

Figure 1. Gravitational wave signals from LIGO’s GW150914 event. 
LIGO team needs to apply some sophisticated noise filtering technique (whitening filter) in order to achieve the signals sought.

The data from LIGO's events was evaluated by Dr. Ulianov, using a new kind of whitening filter that allows a band pass of specific noise sources. This new filter, named the Ulianov Whitening Filter with Noise Band-Pass (UWF-NBP), is basically a standard whitening filter, where the "whitening effect" is canceled out over a small range of frequencies. This means the UWF-NBP's output maintains the same signal generated by a standard whitening filter, but with a "noise signal" (in a given frequency range) added to it.

Section 3 presents the results obtained using the UWF-NBP to process the data recorded from the GW150914 event. The graphs obtained from processing this event show a strange similarity between the signal generated by the collision of two black holes and the $32.5 \mathrm{~Hz}$ noise coming from the Hanford detector. Besides this, the $32.5 \mathrm{~Hz}$ noise level falls exactly when the gravitational wave is detected.

Section 4 presents the results obtained using the UWF-NBP to process the GW151226 event. For this event, the noise level of the output signals (after filtering) is far greater than that of the supposed gravitational waves detected. Furthermore, from the GW151226 event, the medium range frequency (100 to $200 \mathrm{~Hz}$ ) of the gravitational wave template is strongly correlated with the $120 \mathrm{~Hz}$ and $180 \mathrm{~Hz}$ noise sources.

Thus, the UWF-NBP highlights a strong connection between the "gravitational waves" detected and some noise sources, leading to a basic question: As these noise sources are always present in LIGO's detectors, why do these noises only appear in the GW150914 and GW151226 events?

For these authors, the $32.5 \mathrm{~Hz}, 120 \mathrm{~Hz}$ and $180 \mathrm{~Hz}$ noise sources are all connected to the $60 \mathrm{~Hz}$ power source. Thus, some occurrence in the power supply system (in the US power grid) can generate frequency changes in this $60 \mathrm{~Hz}$ signal, affecting these noise sources in a non-periodic manner. This non-periodic noise can avoid LIGO's filter process, appearing to be gravitational wave signals. This point is presented in Section 5, providing an explanation on how two random noises can be observed at same time in both of LIGO's detectors, due to a problem in the DC links that connect the US power grid.

Interestingly, the second of LIGO's detections happened before the first event was published. So, if the LIGO team had data on two gravitational wave events, why did they not present them simultaneously in order to strengthen the evidence of true detection?

The fact is, in the GW151226 event, the level of gravitational waves is lower than the background noise (obtained after applying the whitening filter), making this detection highly questionable.

However, after presenting the first event through the world's media, the LIGO team emerged from a "bleak" period (nearly two decades of activities without any results) to a global celebration, even with the possibility receiving the Nobel Prize in 2016.

Hence, it seems that the LIGO team considered it important to show the second event in mid-2016 in order to ensure its discovery, with two reported detections. 
However, as presented in Section 6, to deal with the GW151226 event's data, the LIGO team changed some procedures. This leads to a change in the GW150914's template (from a real signal, to a complex signal), which also changes its "event time", shifting $18 \mathrm{~ms}$. This change was not notified by the LIGO team, but can be spotted by careful analysis of LIGO's tutorial programs, as detailed in Section 6.

Section 7 presents the conclusion of this article, where it is clear that beyond the theoretical questioning of LIGO's ability to actually detect gravitational waves, there is data pointing to the fact that both of their detections are probably a false alarm.

\section{Theoretical Evidence That LIGO Cannot Detect Gravitational Waves}

In this section we present only a summary of theoretical evidence that LIGO's system cannot detect gravitational waves. More detailed information can be found in articles [4] and [5].

Note that the authors of the articles believe that gravitational waves exist, and fully agree with many theoretical works [6]-[8] that deal with this theme. The point here is that LIGO's system has some serious mistakes, possibly preventing this experiment from detecting gravitational waves.

\subsection{The Michelson Interferometer Cannot Be Used to Detect Gravitational Waves}

According to General Relativity, gravitational waves affect spatial distance, so it simultaneously affects wavelengths of light. Considering this fact, the phase differences of the laser beams propagating inside the arms of Michelson's interferometers, are invariable, while gravitational waves pass through them. Therefore, it was impossible for LIGO to detect gravitational waves by using Michelson's interferometers.

It is important to observe that the LIGO team admits that gravitational waves can change the wavelength of light, so in LIGO's FAQ page [9], they present the following question:

"If a gravitational wave stretches the distance between the LIGO mirrors, doesn't it also stretch the wave-length of the laser light?"

The answer given by the LIGO team is:

"A gravitational wave does stretch and squeeze the wavelength of the light in the arms. But the interference pattern doesn't come about because of the difference between the length of the arm and the wavelength of the light. Instead it's caused by the different arrival time of the light wave's "crests and troughs" from one arm with the arrival time of the light that traveled in the other arm. To get how this works, it is also important to know that gravitational waves do NOT change the speed of light."

These authors consider the LIGO team's answer to be very confusing, showing that they are aware of the problem, but try to avoid it. As presented in [10] this kind of "different arrival time of the light waves" could only occur in the case of two independent laser sources placed in each interferometer arm. As LIGO's detectors use only one laser source (in each interferometer), the interference pattern doesn't occur. 


\subsection{The Results of LIGO's Experiments Go against the Results of Michelson's Experiments}

Before Einstein put forward Special Relativity, Michelson took a dozen years trying to find the absolute motion of the earth, but completely failed. The explanation of SR, for the lack of results with Michelson's experiment, was: when the interferometer rotates, one of the arms contracts (according to the formula of Lorentz transformation), which leads to the speed of light being constant. Thus, the fringe interferences were unchanged.

The method LIGO used is similar to the one used by Michelson. When a gravitational wave hits the interferometer, the length of its arms and the wavelength of the gravitational waves change synchronously. Making a calculation: the speed in which the earth moves round the sun is $30,000 \mathrm{~m} / \mathrm{s}$, so the Lorentz length contraction is $5 \times 10^{-8}$ meters, 5 billion times greater than the gravitational wave strain detected by LIGO's system.

If Michelson did not observe any change in the fringe interferences, then how can LIGO have detected it?

LIGO's experiment was based on a failed experiment and for the same reasons it was also destined to fail.

\subsection{The Formula of General Relativity Was Applied in a Wrong Context}

The formula of General Relativity used to calculate the change of distance is only suitable for particles in a vacuum. The formula is unsuitable for calculating the change in the interferometer's arm lengths, which are fixed on the earth's surface through electromagnetic interaction. Hence, the basis of the calculation in LIGO's experiment is also wrong.

\subsection{Electromagnetic Interaction Makes LIGO's Experiments Impossible}

Electromagnetic force is $10^{40}$ greater than gravity, so gravitational waves can not violate the balance of electromagnetic forces, making LIGO's interferometer vibrate. The reason that LIGO's experiments fail is the same reason why Weber's experiments also did not work. Gravitational waves are too weak to overcome electromagnetic forces between atoms in metal, without any antenna vibration. Therefore, all gravitational wave experiments using interferometers on the earth's surface, including those of LIGO, Virgo, GEO600 and TAMA300, cannot observe gravitational waves.

\subsection{LIGO's Experiments Do Not Verify the Theory of General Relativity}

Even if it is true that LIGO have detected a real signal of gravitational waves (an event of binary black holes merging), this fact does not verify Einstein's theory of gravity. The energy from the black holes merging was transformed into gravitational waves and emitted into the universe's space.

That's all! We cannot say any more.

Thus, the logic used for the LIGO team to confirm Einstein's theories has some problems: At first, based on the data recorded in the laser interferometer and Einstein's 
gravitational theory (a reason), they deduced that there was an event of binary black holes merging, happening in a distant galaxy 1.3 billion years ago (a result). After, based on the so-called consistence between the event and the data observed (a reason), Einstein's gravitational theory becomes verified (a result). Obviously, LIGO's argument is a vicious circle and logically invalid. It is difficult to understand why so many scientists, who signed their names on LIGO's papers, did not know this logical problem.

\subsection{No Singularity Black Holes Were Found}

In paper [11] Dr. Xiaochun points out some mistakes in the Oppenheimer deduction that massive celestial bodies may collapse into singular black holes with infinite densities. This work highlights that the "creation" of singularity black holes, in a star collapsing process, may not be something that actually occurs in nature, at least not in a correct application of the theory of General Relativity. Besides this, until today, not a single black hole with singularity horizon has really been found through astronomical searching. Therefore, LIGO's results are the only proof of the coalescence of binary black holes. However, with so many problems happening in LIGO's experiments, their results are not reliable enough to confirm the existence of singularity black holes.

\subsection{Interferometer's Arm Length Variation Is a 1000 Times Less than Nuclear Radius}

When the laser hits the mirrors in the interferometer's arms, the movement of the mirrors' surfaces caused by the gravitational wave are far less than $10^{-18}$ meters, which in a nuclear atomic scale is hard to be measured. This kind of precision enters into the quantum-scale. Not only is it far beyond the limitation of mankind's technology, it also violates the uncertainty principle ( $\Delta x \cdot \Delta p \sim h$ ), as defined in quantum mechanics.

\subsection{The Method of Numerical Relativity Is Unreliable}

Einstein's equations of gravity are non-linear and difficult to be solved. The method of numerical relativity is put forward to deal with these kinds of problems. However, black holes involve singularities and the law of physics is invalid in singularities. In order to make calculation possible, lots of simplifications had to be introduced, which caused great errors. The boundary and initial conditions have to be reset each time when the computer is near to crashing, so the errors become cumulative. Because Einstein's gravity field equations are non-linear, a "Butterfly effect" can occur and increase the extent of the errors. Hence, the effectiveness of numerical relativity being applied to the collision of two black holes is highly questionable.

\section{The Analysis of the GW150914 Event}

In February 2016, when around two hundred physicists put their name to article [2], which announced LIGO's first detection of a gravitational wave, it is evident that something unusual was happening. LIGO's recorded data, from the GW150914 event, was analyzed for over five months by hundreds of physicists, confirming it to be a true 
gravitational wave detection.

But, if LIGO did indeed make the first observation of a gravitational wave, why did they need such a long analysis involving so many people?

The LIGO team knows that the big problem in their detectors is that the total noise level is a few hundred times higher than the expected gravitational wave level. Besides this, the whitening filter process removes all periodical signals and turns them into white noise. Therefore, the only signals that remain in the whitening filter output are the non periodical signals. As the gravitational waves contain non periodical signals, they also appear in the whitening filter output. This procedure only works optimally if all signals to be removed (noise sources) are always periodic.

But which noise source is only periodic?

Even if we consider a "very stable" noise source (that is periodic in a larger time window), random variations will sometimes occur, changing the level of this noise source in the detector. This means that when a noise source in LIGO's detector has a major variation, for a short time it becomes a non periodic signal, which appears in the whitening filter output.

So, in fact, one single LIGO detector cannot distinguish between a gravitational wave and a non periodic variation in a noise source. Thus, LIGO's gravitational wave detection can only be confirmed when the two detectors receive the same signal in a shorter window than $10 \mathrm{~ms}$. This happens in the GW150914 event, indicating that it can be a genuine detection of gravitational waves, but without actually ensuring that it is not just random noise registered by the two detectors.

The authors downloaded the data from the GW150914 event and used the codes (in Python language) provided by the LIGO team to analyze the recorded signals and obtain the gravitational wave curves, as presented in Figure 1.

It is important to observe that the total noise levels in both detectors are four hundred times greater than the level of the gravitational waves detected in GW150914 event.

This problem becomes evident in Figure 2's curves, which represent the power spectrum density of some signals from LIGO's GW150914 event. In this figure, two big noise sources, with $32.5 \mathrm{~Hz}$ and $60 \mathrm{~Hz}$ are indicated. The black curve represents the power spectrum density of the Numerical Relativity signal (NR strain signal). The red curve represents the output of a whitening filter (matched NR stain signal).

In Figure 2, we can see that the whitening filter process removes the power spectrum near to $35.5 \mathrm{~Hz}$ and $60 \mathrm{~Hz}$ from the NR strain signal. Thus, the matched NR stain signal becomes a poor representation of the Numerical Relativity signal.

Figure 3 presents the same signals (NR strain in green and matched NR in red) in the time domain, where we can see that the Numerical Relativity signal has some seconds of duration, but after the whitening filter process, the matched NR signal has less than 0.05 seconds of duration.

Due to the large number of noise sources present in LIGO's detectors, in order to identify if a gravitational wave was detected, the LIGO team applied two different types of searches: 


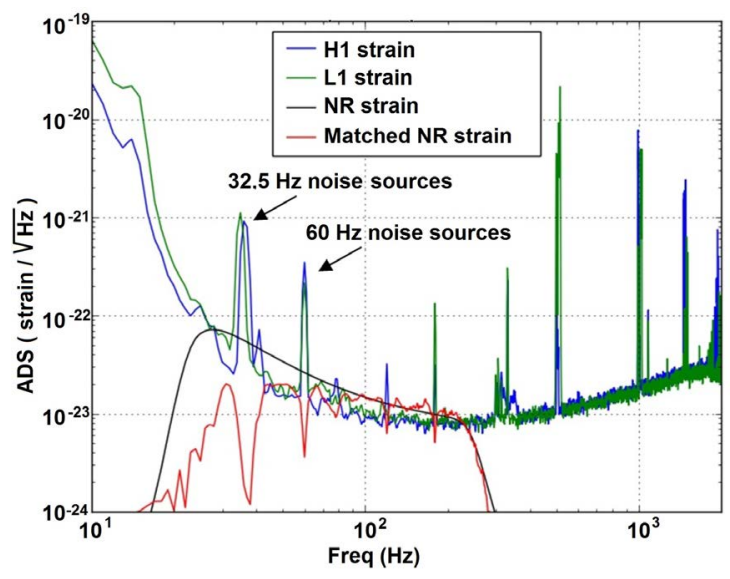

Figure 2. Power spectrum density of signals from LIGO’s GW150914 event.

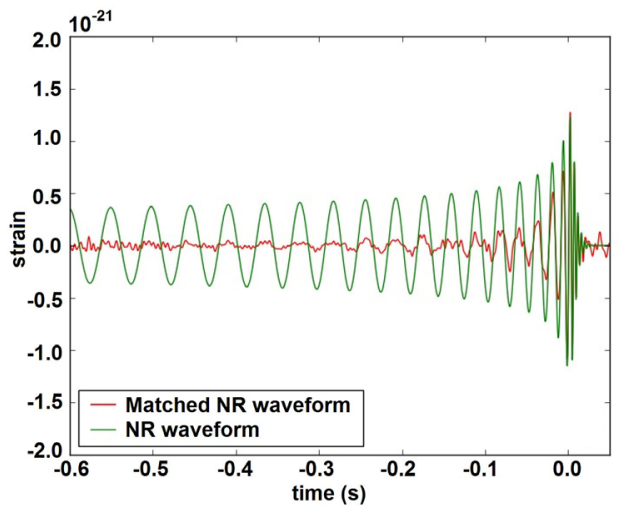

(a)

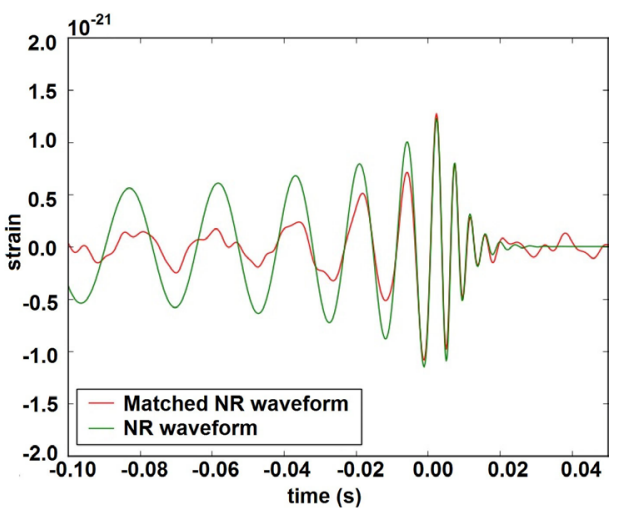

(b)

Figure 3. Numerical relativity signal (in green) and matched NR signal (in red): (a) Signals at 0.6 $\mathrm{s}$ time window; (b) The same signals at $0.1 \mathrm{~s}$ time window.

- Whitening filter search: This search uses a whitening filter [12] to process the recorded data. This kind of filter basically transforms periodic signals into white noise, so only the signals that are non-periodic, pass through the filter. The main problem with the whitening filter search is that the gravitational waves also have some periodic signals that are removed by the filter.

- Optimal matched filtering search: This search uses thousands of templates (waveforms), predicted by General Relativity [13], testing each one to see if it is present in the recorded data in one certain time window (usually 16 or 32 seconds). The main problem with this kind of search is not giving results as gravitational wave curves (like those given by the whitening filter). The optimal matched filtering only indicates if one tested template is present in the recorded data, given as an SNR (Signal Noise Relation) curve, where the curve peak indicates the template waveform position.

The Ulianov Whitening Filter with Noise Band-Pass was applied to LIGO's data recorded in the GW150914 event to analyze the noise source from 31.5 to $43.5 \mathrm{~Hz}$, presented in Figure 2 as the $32.5 \mathrm{~Hz}$ noise source. 
Figure 4 presents the results of processing LIGO's Hanford data, using a standard whitening filter (in green) and the UWF-NBP (in red). In this figure, it is easy to see that the $32.5 \mathrm{~Hz}$ noise appears clearly in the UWF-NBP filter's output, showing that this new filter works.

Figure 5 presents the $32.5 \mathrm{~Hz}$ noise in a larger time window. In this figure, it is easy to observe that the $32.5 \mathrm{~Hz}$ noise source is modulated by sinusoid, with a frequency near to $2.5 \mathrm{~Hz}$, presented as a black curve in Figure 6 .

This means that the $32.5 \mathrm{~Hz}$ noise is generated by some kind of oscillator, with a resonance frequency close to $32.5 \mathrm{~Hz}$, which is "powered" by a $30 \mathrm{~Hz}$ signal, resulting in a low frequency modulation $(2.5 \mathrm{~Hz}=32.5 \mathrm{~Hz}-30 \mathrm{~Hz})$. Obviously, this $30 \mathrm{~Hz}$ "power source" is a sub-harmonic signal from the $60 \mathrm{~Hz}$ power source grid.

Figure 7 is basically the same curve from Figure 4, but now the $32.5 \mathrm{~Hz}$ noise level is presented for the time before the GW150914 event (black line) and after it (blue line). If

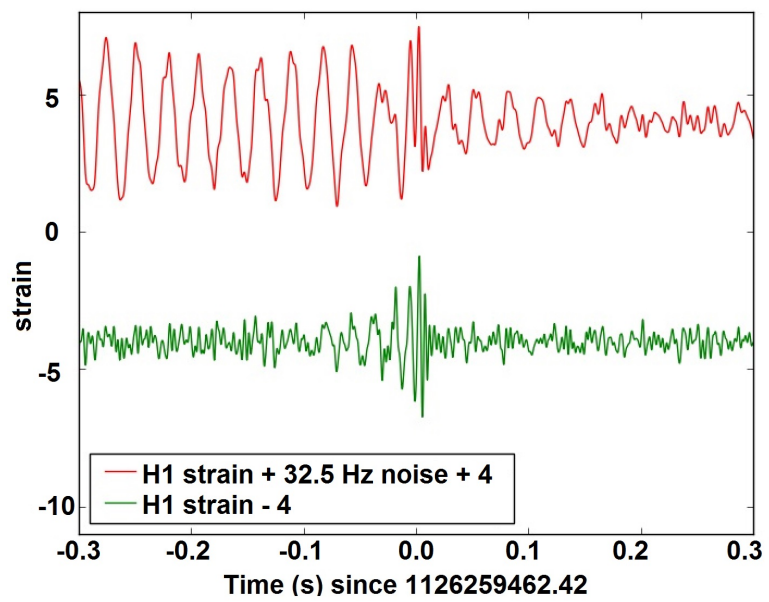

Figure 4. Curves obtained by processing the data recorded from Hanford (H1 strain), with the standard whitening filter (in green) and the UWF-NBP (in red). The signals are added to an offset value in order to appear separately.

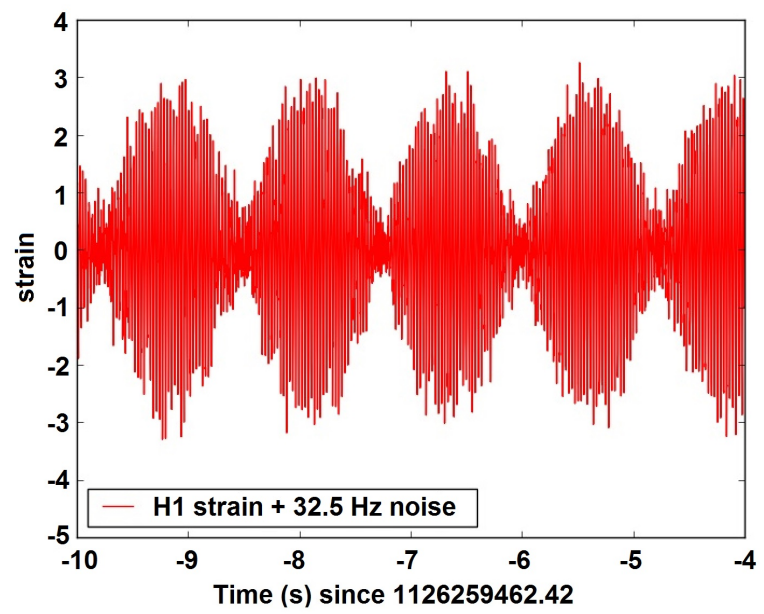

Figure 5. Hanford's $32.5 \mathrm{~Hz}$ noise source. 


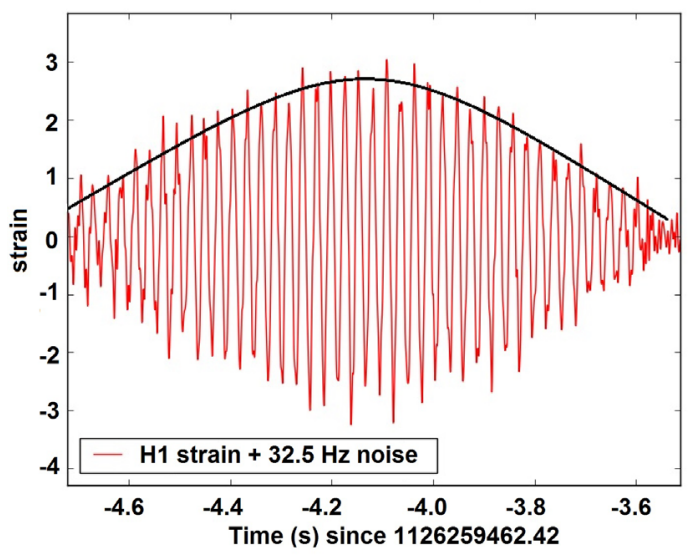

Figure 6. Hanford's $32.5 \mathrm{~Hz}$ noise source (in red) modulated by a sinusoid (in black), with frequency of $2.5 \mathrm{~Hz}$.

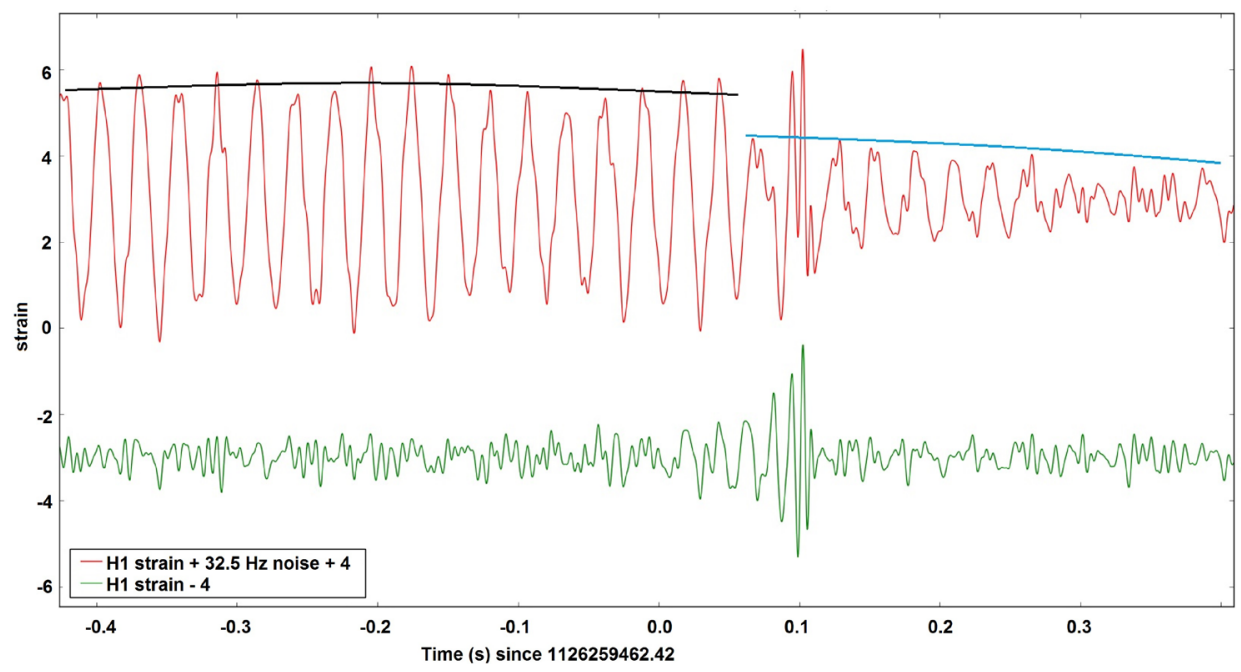

Figure 7. The same curves from Figure 4, observing the $32.5 \mathrm{~Hz}$ noise source level.

we compare the $32.5 \mathrm{~Hz}$ noise level in Figure 6 (the continuous curve in black) with the $32.5 \mathrm{~Hz}$ level in Figure 7 (the two curves in black and blue), it is easy to see that something has affected the noise level near time zero, exactly at the point where LIGO detects the end of the gravitational waves. This fact leads us to question:

How can a "gravitational wave" that hits the detector, affect the $32.5 \mathrm{~Hz}$ noise level?

The LIGO team calculated the Numerical Relativity (NR) signal, a gravitational waveform generated from the GW150914 event, which is supposedly connected to the collision of two black holes. Moreover, there is a great similarity between the NR signal and the $32.5 \mathrm{~Hz}$ noise source, as presented in Figure 8. In this figure, the black signal is the NR signal multiplied by the factor of $4 \times 10^{21}$ (necessary in order to keep the same scale, as the whitening filter multiplies the input signal by this factor) and the signal in red is the $35.5 \mathrm{~Hz}$ noise, obtained from the UWF-NBP's filter.

Comparing the NR signal and the $32.5 \mathrm{~Hz}$ noise signal, presented in Figure 8, leads us to question: 


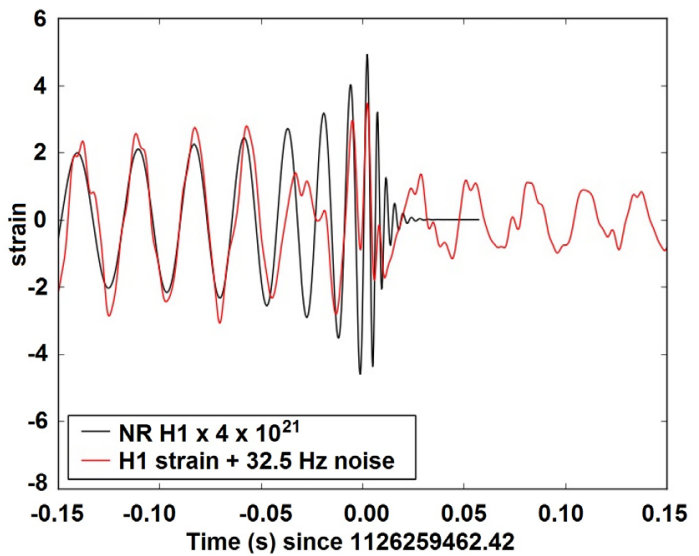

Figure 8 . The curve of the NR signal multiplied by $4 \times 10^{21}$ (in black) and the $32.5 \mathrm{~Hz}$ noise (in red).

Why is the NR signal, which is generated from the collision of two black holes, so similar to the $32.5 \mathrm{~Hz}$ noise that continually comes from LIGO's detector?

Why are these two signals exactly synchronized in time?

These questions point to a connection between the NR signal and the $32.5 \mathrm{~Hz}$ noise in the Hanford detector, raising strong doubts as to whether LIGO have actually detected a gravitational wave in the GW150914 event.

\section{The Analysis of the GW151226 Event}

The data from the GW151226 event was also made available on the internet by the LIGO team, with some programs (in Python language) that enabled the plotting of the curves in Figure 9.

In Figure 9 we can see that the whitening filter search completely fails, because the level of the gravitational waves' curves (in black) is lower than the white noise level (in red and green). Thus, in the GW151226 event, the gravitational wave curves were obtained by LIGO, using only the optimal matched filtering search.

Figure 10 presents the SNR curve obtained by using the optimal matched filter, based on the gravitational wave template (the black curves in Figure 9).

To better evaluate the optimal matched filter's results, the same gravitational wave template was shifted by 3 seconds, defining the "GW Sim" signal, presented in Figure 11. This signal was added to LIGO's recorded data (from both detectors) in the GW151226 event. After, the optimal matched filter was used to generate a new SNR curve, presented in Figure 12.

As expected, in Figure 12, we can see two peaks in the SNR curve, one from the real event (at zero time) and another from the simulated signal (at $3 \mathrm{~s}$ time).

The curves in Figure 12 show that the optimal matched filter can detect the gravitational wave template, with the SNR near to 10. But, what happens if the simulated signal is slightly different?

To answer this question, we need to define two new "GW Sim" signals, presented in Figure 13 in blue. They are basically the same as the curves of the gravitational wave 

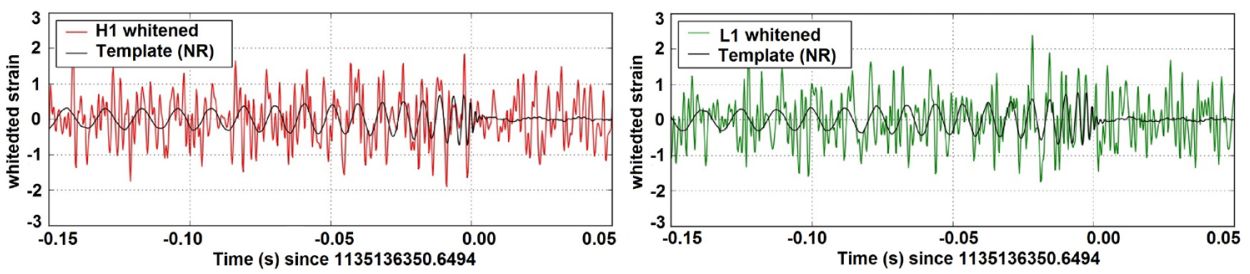

Figure 9. Gravitational wave signals detected in the GW151226 event.
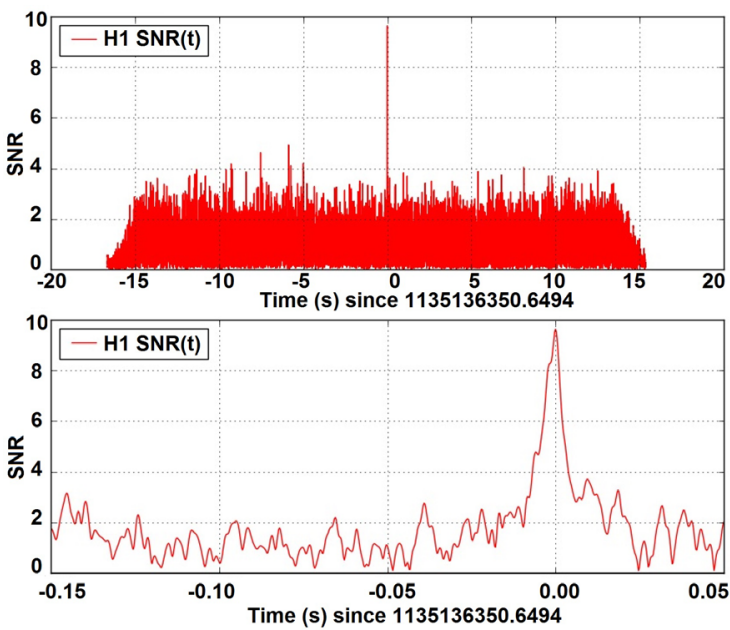

Figure 10. SNR curve in the GW151226 event in the Hanford detector.

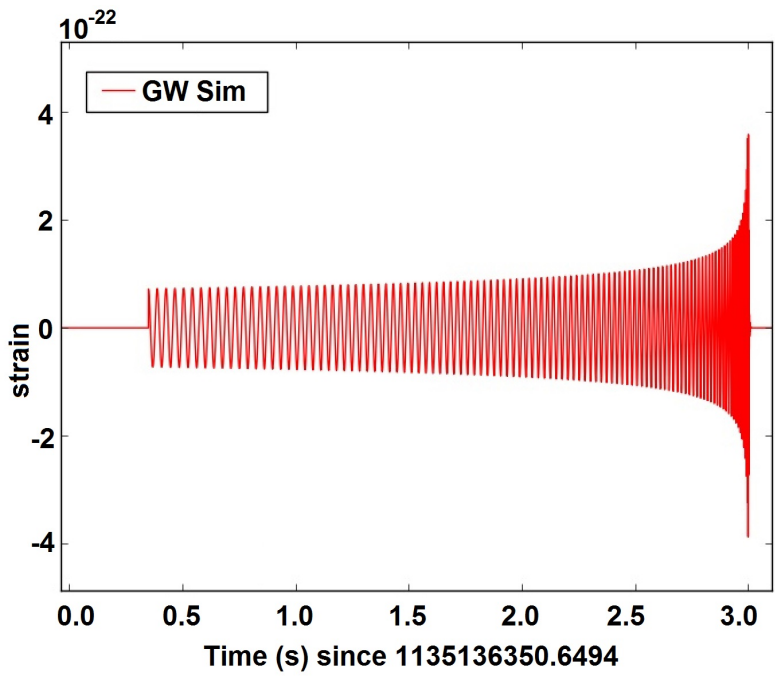

Figure 11. Simulated signal added to LIGO's recorded data in the GW151226 event.

template, with a band-pass filter applied and multiplied by a constant factor, in order to maintain the same power level.

The signal presented in blue in Figure 13(a) was added to LIGO's recorded data and processed with the optimal matched filter, generating the SNR curve, presented in Figure 14.

The signal presented in blue shown in Figure 13(b), was also added to LIGO's re- 

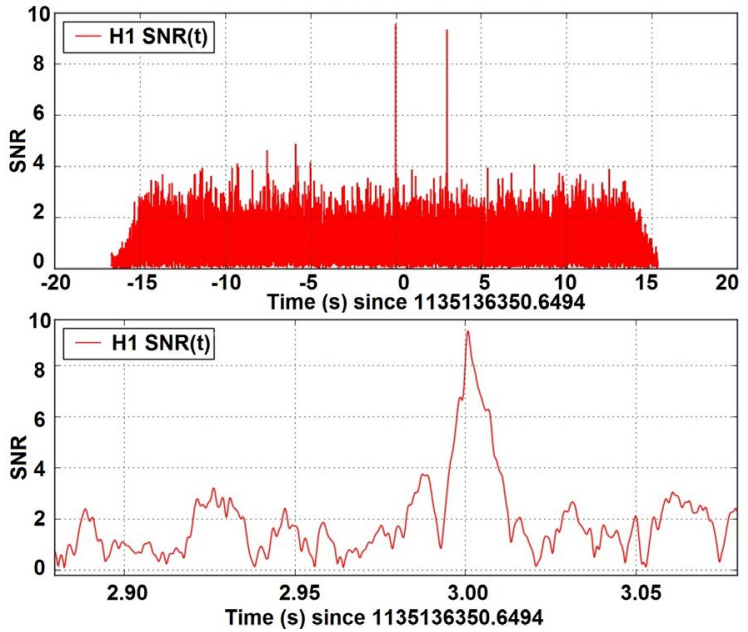

Figure 12. SNR curves obtained by processing the simulated signal.

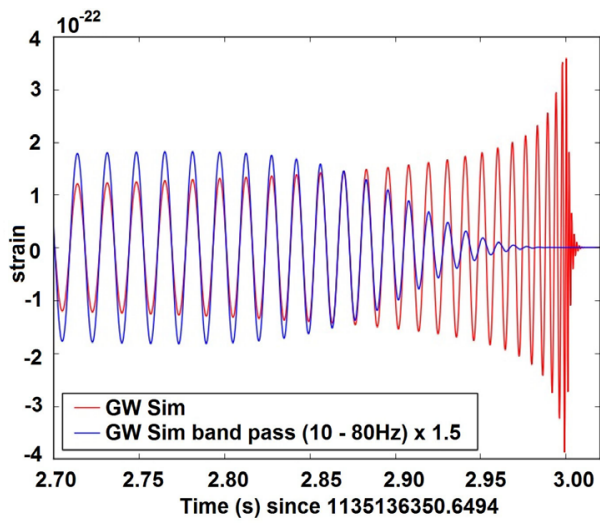

(a)

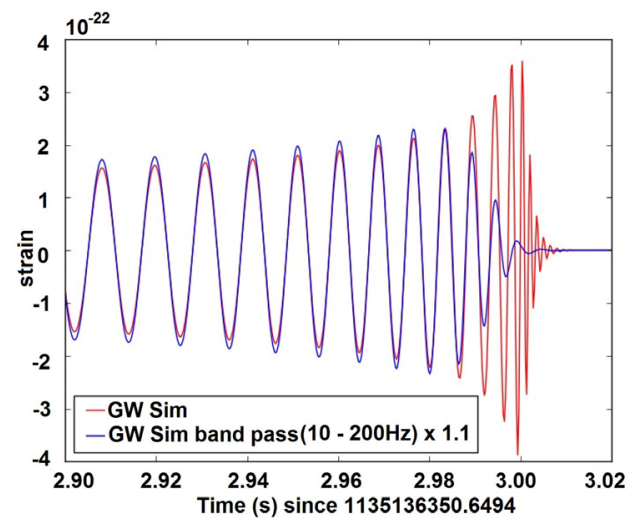

(b)

Figure 13. Two new simulated signals: (a) Band-pass filter (from 10 to $80 \mathrm{~Hz}$ ) applied and multiplied by 1.5; (b) Band-pass filter (from 10 to $200 \mathrm{~Hz}$ ) applied and multiplied by 1.1 .
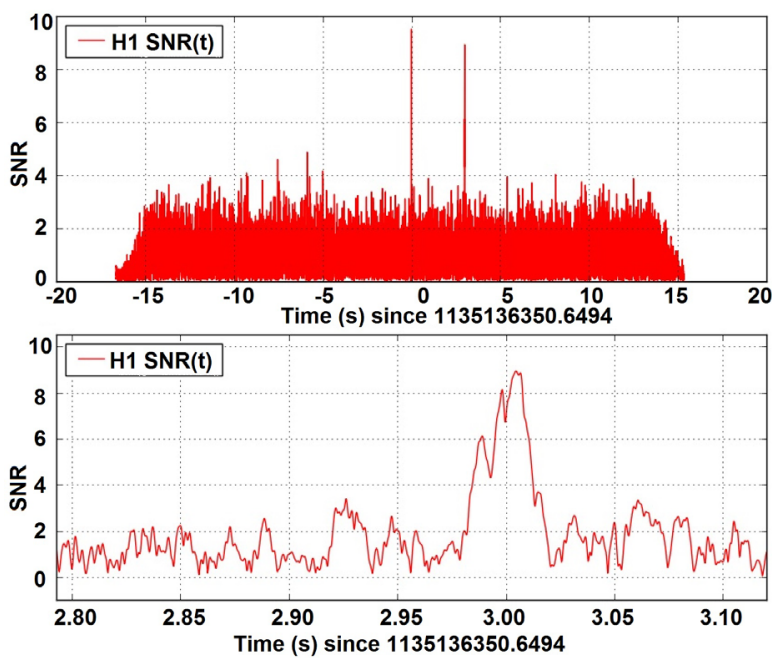

Figure 14. SNR curves obtained by processing the simulated signal presented in blue, shown in Figure 13(a). 
corded data and processed with the optimal matched filter, generating the SNR curve, presented in Figure 15.

The SNR curve in Figure 15 indicates that if the blue signal in Figure 13(b) is present at zero time, the SNR value in the optimal matched filter output is also near to 10. This means that while LIGO may have detected the gravitational wave template (the red curve in Figure 13(b)), it would also have detected the "GW Sim" template presented in blue in Figure 13(b).

Figure 16 presents two new curves: The red curve is the same gravitational template (the red curve in Figure 13(b)) that was processed by a band-pass filter (from 100 to $200 \mathrm{~Hz}$ ). The blue curve is the result of adding the $120 \mathrm{~Hz}$ noise source to the $180 \mathrm{~Hz}$ noise source. These noise signals are obtained by applying the UWF-NBP filter to the data recorded in the Hanford detector in the GW151226 event.
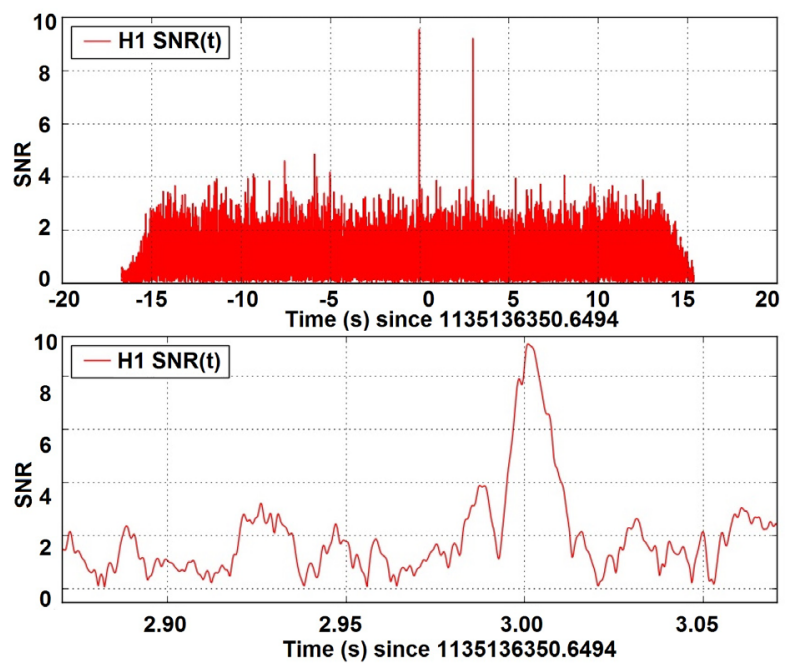

Figure 15. SNR curves obtained by processing the simulated signal presented in blue, shown in Figure 13(b).

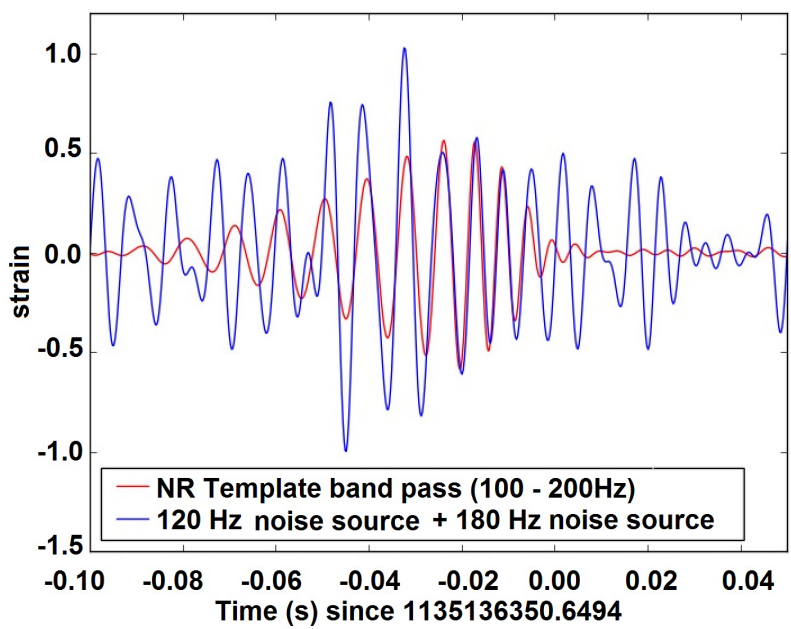

Figure 16. Gravitational wave template processed by a band-pass filter (100 to $200 \mathrm{~Hz}$, in red) and $120 \mathrm{~Hz}$ noise added to the $180 \mathrm{~Hz}$ noise (in blue). 
The curves in Figure 16 provide clear evidence that the GW151226 gravitational wave template (found by the optimal matched filter) has a medium range frequency ( 100 to $200 \mathrm{~Hz}$ ) that is connected to the $120 \mathrm{~Hz}$ and $180 \mathrm{~Hz}$ noise sources that appear in the Hanford detector. Note that these kinds of curves are also obtained in the Livingstone detector's recorded data in the GW151226 event.

This means that the optimal matched filter did not detect a complete template, but in fact, may have detected the variation of frequency in the template's medium range. Therefore, some event in the US power grid that slightly modifies the $60 \mathrm{~Hz}$ frequency (and its $120 \mathrm{~Hz}$ and $180 \mathrm{~Hz}$ harmonics) can generate a template match (in the optimal matched filter's search), thus, giving a high SNR value. Evidence that the optimal matched filter is picking up some variations in noise sources can be seen in Figure 17.

If we look at the SNR values in Figure 10 again, as presented in Figure 17, we can note that the optimal matched filter found the same gravitational wave template at -5.87 s time with an SNR equal to 5 .

This means that if the LIGO team had found a gravitational wave with an SNR equal to 10 in the GW151226 event at zero time, they would also have found the same gravitational wave with an SNR equal to 5 at -5.87 s. However, this was impossible because no gravitational wave had occurred at this earlier time. This is further strong evidence, that the optimal matched filter detected something else at $-5.87 \mathrm{~s}$, which was probably also caused by a change in the $60 \mathrm{~Hz}$ frequency in the US power grid.

Besides this, we can question: What does an SNR equal to 10 really mean (indicating a gravitational wave detection), if in the same data we find a false gravitational wave detection, with an SNR equal to 5?

Obviously, an SNR value obtained in a time when there is only noise should, at most, be equal to one. Thus, an SNR value of 5 (where there is no signal) indicates some error in the SNR calculation made by the LIGO team. As the SNR calculation gives a normalized curve (multiplied by a constant), we can consider that the "true" SNR value is multiplied by some factor that, in GW151226 event, is at least equal to 5. So, dividing
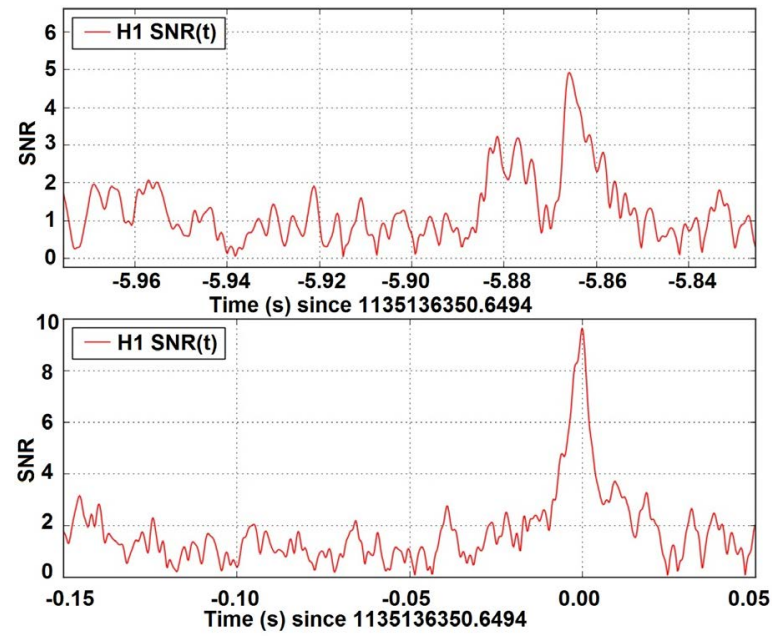

Figure 17. Same SNR curves from Figure 10 in another time window for the upper graph. 
the SNR curve by 5, the maximum SNR value (when there is only noise), becomes equal to 1 , which is an acceptable value. Thus, for the GW151226 event, the SNR value equal to 10 becomes a true SNR value, equal to 2 .

Applying this procedure in the GW150914 event, the SNR value equal to 24 (as indicated by the LIGO team) becomes a true SNR value, equal to 4.8 .

Note that as the SNR values are used to calculate the "false alarm" rates in both events, a low SNR true value gives a lower false alarm rate.

\section{The Variations of Frequency in the US Power Grid}

The US power grid configuration is presented in Figure 18, which shows two large interconnection grids: Eastern interconnection and Western interconnection. Four DC links (red circles) connect the Eastern Grid with the Western Grid. These DC links are important because they allow the transmission of power from one system to another, optimizing the generation of electricity.

An abrupt change of energy flow in the DC links can generate frequency instabilities in these two interconnections. For example, if the Eastern interconnection has more energy generation than its consumption, and the Western interconnection has more consumption than it generates, these DC links can be used to transfer power from the Eastern grid to the Western one. In this case, if some of the DC links open (due to an operational problem), the Eastern grid generators will become momentarily unloaded and tend to rotate faster. At the same time, the Western grid's generators will become momentarily overloaded and will tend to rotate slower. In a few seconds, the generator's controls will act (for example, by varying the angle of the hydroelectric turbine's blades, or changing the fuel injection in some of the thermoelectric generators), changing the total active power generated in each grid. This means that a DC link event will generate a small $60 \mathrm{~Hz}$ frequency change over the whole US power grid in the same time window.

The LIGO system monitors the fluctuations of the power grid [14], using "power voltage monitors" that are installed in the electronics room of each building.

Thus, LIGO's power monitoring did not detect the $60 \mathrm{~Hz}$ frequency variations of the power grid, because the voltage level did not change.

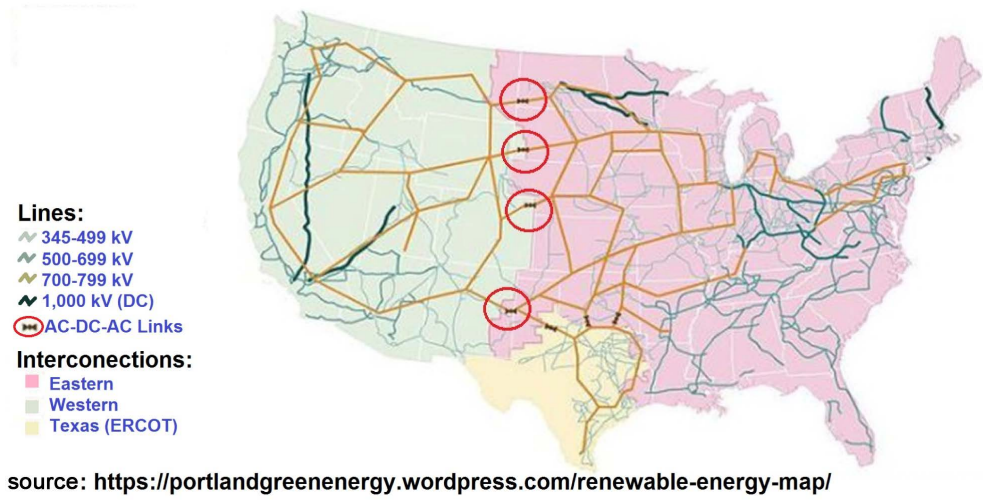

Figure 18. US Power Grid. Four DC links (red circles) connect Eastern Grid and Western Grid. 
For example, a small change in the $60 \mathrm{~Hz}$ frequency (e.g. from $60.1 \mathrm{~Hz}$ to $59.9 \mathrm{~Hz}$ ) can cause a notable phase shift in the $32.5 \mathrm{~Hz}$ noise source (e.g. a $0.2 \mathrm{~Hz}$ variation, causing a time shift of $0.032 \mathrm{~s}$ ). This produces a transitory signal that may be related to the high frequency signals of a $0.05 \mathrm{~s}$ duration, recorded by the LIGO team in the GW150914 event, which is associated with a gravitational wave signal.

Furthermore, variations in the $60 \mathrm{~Hz}$ frequency (that generate the variations in the $120 \mathrm{~Hz}$ and the $180 \mathrm{~Hz}$ noise sources) may also have been detected as a gravitational wave template, by LIGO's optimal matched filtering search in the GW151226 event.

\section{LIGO's "Little Trickery" in the GW150914 Event}

The analysis presented in this article is based on information provided by the LIGO team, including several papers, tutorials, data recorded from the GW150914 and GW151226 events and the Python programs used to process the data.

LIGO provides two tutorial programs that process the data recorded from the GW150914 event:

- GW150914_tutorial.py: This python code [15] was originally developed to be applied to just the GW150914 event's recorded data. The code uses the whitening filter, but without using the optimal matched filtering search.

- LOSC_Event_tutorial.py: This python code [16] was presented to deal with the GW150914 and GW151226 events' recorded data. As the whitening filter does not work in the GW151226 event, this new code also uses the optimal matched filtering search.

- Hence, in order to validate the GW151226 event's data, the LIGO team generated a new type of process that must also be valid for all past events. However, from thorough analysis of the GW150914 event, some problems were found when using the two python programs.

- The event time (time where the gravitational wave was detected) used as the input parameter for each program was changed: In the GW150914_tutorial.py program the event time is defined in code (tevent $=1126259462.422$ ). In the LOSC_Event_ tutorial.py program the event time is read from a data file (O1_events.json that contains: "tevent": 1126259462.44). Thus, the event time was changed by $18 \mathrm{~ms}$, a large value, considering that $10 \mathrm{~ms}$ is the maximum time window for simultaneous detection of both detectors. It is important to observe that the LOSC_Event_tutorial.py program can calculate the event time. Therefore, the Hanford event time is equal to $1126259462.4414 \mathrm{~s}$ and the Livingston one is equal to $1126259462.4343 \mathrm{~s}$, with the difference between them also being $7 \mathrm{~ms}$.

The Numerical Relativity (NR) signal used in these programs was also changed: In the GW150914_tutorial.py program the NR signal is defined by the NR_H1 variable, which is a vector with real data that is read from a text file

(GW150914_4_NR_waveform.txt). In the LOSC_Event_tutorial.py program the NR signal has complex numbers that are defined by two vectors (template_c and template_p), read from an hdf5 file (GW150914_4_template.hdf5). Figure 8 presents these 
two kinds of NR signals, where it is easy to observe that when the time is near to zero, the signals are synchronized. Nevertheless, for the initial times (negative time values) the signals are shifted. Obviously, the real part (green signal) and the imaginary part (blue signal) of the complex template should be 90 degrees out of phase. But, if the NR signal used in both programs represents the same gravitational wave, the NR_H1(red signal) signal peaks are expected to be somewhere in the middle of the red and green curves, as indicated in the red text in Figure 19.

To publish the data and programs associated with the second gravitational wave detection (the GW151226 event), the LIGO team had to make a new program to accommodate the GW151226 event's results. But this new program also needs to be applied to processing the GW150914 event's data, as it would be absurd for the LIGO team to use a new program for processing each new gravitational wave detection. Therefore, this new program (the LOSC_Event_tutorial.py, developed to process the GW151226 event) needs to be used to deal with all events.

Thus, the LIGO team does a little "trickery" in the GW150914 event tutorial program, slightly changing the shape of the NR signal (from a real template to a complex template, as presented in Figure 19) and this modifies the event's zero time ("tevent" input parameter), shifting its value by $18 \mathrm{~ms}$. The LIGO team can explain that the complex template is better and does not affect the relative time in which the gravitational wave hits the two detectors ( $7 \mathrm{~ms})$. However, they changed the GW150914 event's initial time by $18 \mathrm{~ms}$ in the new tutorial program, without any warning.

Besides this, article [2] presents some gravitational wave curves (see Figure 20) and indicates that: "the shaded areas show $90 \%$ credible regions for waveform reconstructions". Though, in the GW150914 event, if we change the template from the real NR signal (red curve in Figure 19, the same as the red curve presented in Figure 20) to the complex template (the blue and green curves in Figure 19, that occupy a different position in time), the gravitational wave curve will not be inside the shaded area shown in Figure 20 (surrounding the red signal). Thus, the LIGO team claiming "the

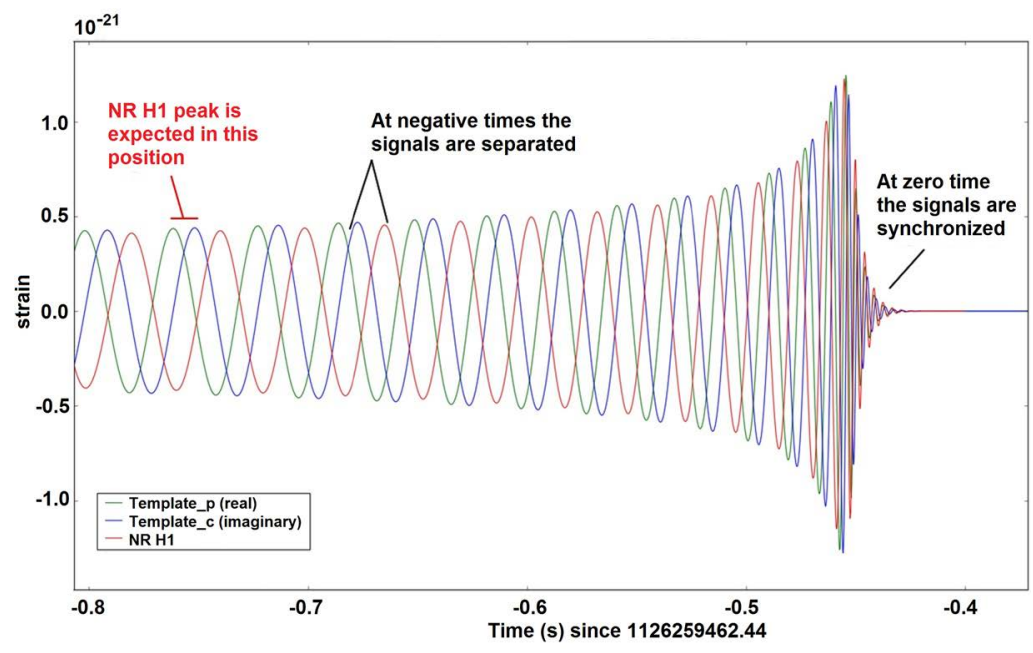

Figure 19. NR signals associated to the GW150915 event. 


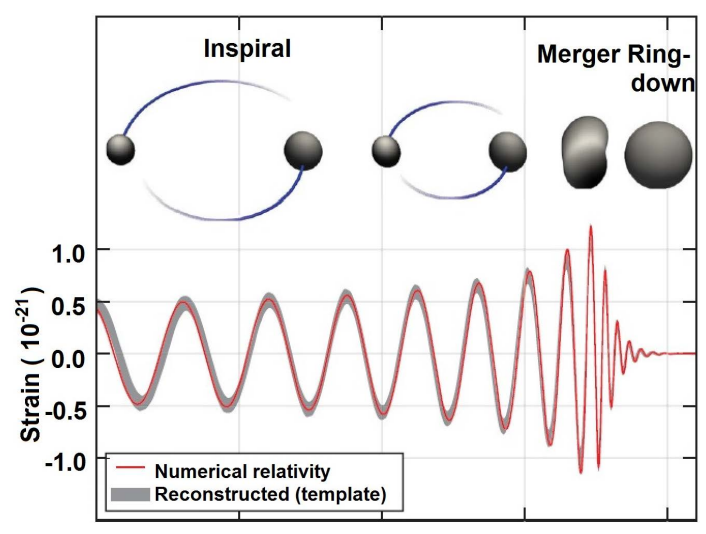

Figure 20. Figure showing NR signal presented in article [2].

shaded areas show $90 \%$ credible regions" is a con or a lie, because the new gravitational wave template (created by the LIGO team in the LOSC_Event_tutorial.py code and also processing the GW151226 event) comes totally out of these shaded areas.

\section{Conclusions}

In article [2], the first gravitational wave detection in the GW150914 event was confirmed by a statistical assumption:

"Measured on a background equivalent to over 67,400 years of data and including a trials factor of 3 to account for the search classes, its false alarm rate is lower than 1 in 22,500 years".

Besides this, the "false alarm rate" does not consider that an external cause can affect both of LIGO's detectors in the same time window. Knowing that the noise sources in both detectors are strongly connected to the $60 \mathrm{~Hz}$ power grid, it is evident that variations in the $60 \mathrm{~Hz}$ frequency can affect some noise sources.

As presented in Section 5, an event in the US power grid's DC Links can change the $60 \mathrm{~Hz}$ frequencies in the Eastern interconnection and Western interconnection, affecting both of LIGO's detectors at the same time. As LIGO's monitoring system uses "power voltage monitors" [14], it did not detect any $60 \mathrm{~Hz}$ frequency variations.

It is clear and evident that LIGO's detectors have at least one source of problems (not observed by LIGO's monitoring system), which can affect both detectors simultaneously, generating a false detection of gravitational waves. This evidence alone is enough to invalidate the "false alarm rate lower than 1 in 22,500 years", denying the statistical evidence that LIGO has detected gravitational waves in the GW150914 event.

Furthermore, the analysis of some noise sources, presented in Sections 2 and 3, using the Ulianov Whitening Filter with Noise Band-Pass, points out some important results: the gravitational waves' "shapes" are very similar to some noise sources' "shapes", with the signals perfectly synchronized in time; the $32.5 \mathrm{~Hz}$ noise source level changes exactly when the gravitational waves are detected in the GW150914 event.

As a gravitational wave cannot be so similar to the noise present in the detectors and a cosmic event cannot affect the level of a noise source in the detector, it is evident that 
both detections of the gravitational waves carried out by LIGO in 2015 are false alarms.

For these authors, it is clear that "Advanced LIGO" has improved the LIGO system, making the detectors ten times more sensitive, but this improvement has put the detection range lower than the remaining noise level (after using the filtering techniques available).

Hence, the first action LIGO's managers need to do is improve its power grid monitoring system, observing variations in the $60 \mathrm{~Hz}$ frequency, which is a possible source of false gravitational wave detections, which today is not being observed.

Secondly, the Ulianov Whitening Filter with Noise Band-Pass is a new tool that can be used by the LIGO team to better understand the noise sources in the detectors and can help avoid false detections.

Thirdly, a broad discussion on the theoretical bases currently used by LIGO should be held. There are many people questioning the LIGO system, whose thoughts should be heard in some kind of workshop open to the scientific community.

And finally, there are some alternatives for detecting gravitational waves, such as, using the time dilation phenomenon [17]. Since time is much less subject to external interferences, this new type of detector [10] may be able to observe low frequency gravitational waves, with periods in the range of seconds, minutes or even hours.

\section{References}

[1] Weber, J. (1960) Physical Reviews, 117, 306-313. http://dx.doi.org/10.1103/PhysRev.117.306

[2] Abbott, B.P., et al. (2016) Physical Review Letters, 116, Article ID: 061102. http://dx.doi.org/10.1103/physrevlett.116.061102

[3] Abbott, B.P., et al. (2016) Physical Review Letters, 116, Article ID: 241103. http://dx.doi.org/10.1103/physrevlett.116.241103

[4] Ulianov, P.Y. (2016) Global Journal of Physics, 4, 104-421.

[5] Mei, X.C. and Yu, P. (2016) Journal of Modern Physics, 7, 1098-1104. http://dx.doi.org/10.4236/jmp.2016.710098

[6] Hong, L.F. (2014) Optical Resonant Cavity and Detection of Gravitational Waves. Science Publishing Company, Singapore, 239, 246, 331.

[7] Ohanian, H.C. and Ruffini, R. (1994) Gravitation and Space-Time. WW Norton \& Company Inc., New York, 155.

[8] Yun, J.W. and Xing, J.Z. (2014) Advance in Astronomy, 32, 349.

[9] LIGO. Frequently Asked Questions. https://www.ligo.caltech.edu/page/faq

[10] Ulianov, P.Y. (2016) Global Journal of Physics, 4, 281-300.

[11] Mei, X.C. (2014) International Journal of Astronomy and Astrophysics, 4, 656-667. http://dx.doi.org/10.4236/ijaa.2014.44060

[12] Abbott, B., et al. (2016) Observing Gravitational-Wave Transient GW150914 with Minimal Assumptions. http://arxiv.org/pdf/1602.03843.pdf

[13] Usman, S.A., et al. (2016) An Improved Pipeline to Search for Gravitational Waves from Compact Binary Coalescence. http://arxiv.org/abs/1508.02357

[14] Abbott, B.P., et al. (2016) Characterization of Transient Noise in Advanced LIGO Relevant 
to Gravitational Wave Signal GW150914. arXiv: 1602.03844.

[15] LIGO. GW150914 Event Tutorial Python Code. https://losc.ligo.org/s/events/GW150914/GW150914_tutorial.zip

[16] LIGO. GW141226 Event Tutorial Python Code. https://losc.ligo.org/s/events/LOSC_Event_tutorial.zip

[17] Cahill, R.T. (2006) Progress in Physics, 3, 60-65.

Submit or recommend next manuscript to SCIRP and we will provide best service for you:

Accepting pre-submission inquiries through Email, Facebook, LinkedIn, Twitter, etc. A wide selection of journals (inclusive of 9 subjects, more than 200 journals)

Providing 24-hour high-quality service

User-friendly online submission system

Fair and swift peer-review system

Efficient typesetting and proofreading procedure

Display of the result of downloads and visits, as well as the number of cited articles

Maximum dissemination of your research work

Submit your manuscript at: http://papersubmission.scirp.org/

Or contact jmp@scirp.org 